\title{
«Je souhaite mourir à 75 ans» - vraiment?
}

\section{Jean Martin}

Dr med., membre de la rédaction, ancien membre de la Commission nationale d'éthique

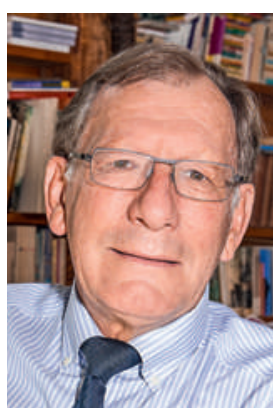

Ezekiel Emanuel, 58 ans, est un oncologue connu aux USA dans le domaine de la bioéthique, issu d'une famille de brillants sujets (son frère Rahm a été conseiller du président Obama et est maire de Chicago). Il était à Genève début juin, parlant du thème qu'il a traité dans The Atlantic, grand magazine de la Côte Est des USA [1]. «Notre espérance de vie a fortement progressé. Il s'agit certes d'un progrès mais cet allongement de la vie s'est accompagné d'une augmentation du nombre d'années vécues avec des handicaps.» Correct: nos homes hébergent beaucoup de vieilles personnes (très) dépendantes. Mais ne pas oublier non plus comment les avancées de l'orthopédie ou de la cardiologie interventionnelle, par exemple, procurent encore à des gens âgés une bonne qualité de vie.

Cette évolution vers le moins, et le moins efficace, est l'occasion d'une réflexion sereine, d'une bénéfique prise de recul.

«Nous avons à faire face à des limitations physiques et mentales, nos attentes diminuent. Sans choix conscient, nous ne remarquons pas que nous n'avons plus guère d'aspirations.» Je comprends bien cela chez quelqu'un marqué par le Rêve américain, mais d'autres (dont j'espère être) pensent que cette évolution vers le moins, et le moins efficace, est l'occasion d'une réflexion sereine, d'une bénéfique prise de recul.

«Chose importante: quel souvenir voulons-nous laisser? Nous ne voulons pas qu'on se souvienne de nous comme des fardeaux. A 75 ans, nous atteignons ce moment unique où nous avons vécu une vie riche et avons, on peut l'espérer, apporté à nos enfants et petits-enfants des choses dont ils garderont la mémoire; plus tard, ces souvenirs de grande vitalité vont être poussés de côté par les manifestations (the agonies) du déclin et nos besoins croissants de soins et d'aide.»

Comment, alors, Emanuel entend-il faire? «A partir de 75 ans, je devrai avoir une vraiment bonne raison pour voir un médecin. Je n'accepterai plus de traitements curatifs, seulement des palliatifs. Je ne voudrai plus de tests préventifs. Donc, plus de colonoscopies ou autres dépistages. Je ne serai pas intéressé par un résultat de PSA. Plus d'épreuve cardiaque d'effort, plus de pacemaker ni de défibrillateur implantable», etc.
«Ce que j'aimerais, dit-il à Le Temps [2], c'est que les gens s'interrogent sur ce qu'ils souhaitent pour leur fin de vie. Si on y réfléchissait vraiment, seul un petit nombre d'entre nous souhaiteraient arriver jusque là.» Là, nous sommes entièrement d'accord. Mais sur l'approche à adopter? Il semble tomber sous le sens que l'attitude qu'il prône ne va pas beaucoup diminuer les situations d'inconfort et de dépendance avant de mourir, les périodes pénibles pour la personne comme pour ses proches. Ou imagine-t-il que, par son refus des antibiotiques notamment, la formule de nos prédécesseurs, «la pneumonie est l'amie des grands vieillards», va trouver une nouvelle jeunesse (si on peut dire!). Chez nous en tout cas, il y a déjà une retenue devant les traitements héroïques maximalistes. Mais il est vrai qu'aux Etats-Unis, Emanuel le relève, l'«obligation technologique» (de tout faire) reste un écueil d'importance.

Ce qui interpelle, c'est que cet auteur s'exprime depuis des années contre le suicide assisté ou l'euthanasie. Pour ma part, je ne suis pas membre d'Exit tout en étant ouvert à la problématique. Je peux imaginer que l'assistance au suicide ne se passe pas toujours de manière optimale mais j'entends des témoignages dans le sens que, au lieu d'être forcément un évènement dur, déchirant, elle peut être une occasion de dialogue voire de réconciliation, de bilan marqué par la sérénité (ceci entre autres parce qu'une date est fixée pour la fin). Et, comme beaucoup, j'apprécie la notion que, en cas de dépendance et souffrance irréversible, je pourrais obtenir une aide à mettre un terme à mon existence. Alors que, dans l'approche d'Emanuel, tout en ne voulant plus de mesures efficaces à intention curative, on attend que son propre état se dégrade totalement. Peu riante perspective.

Dernières phrases de notre confrère [1]: «75 ans est tout ce que je souhaite vivre. Mes filles et mes amis vont continuer à essayer de me convaincre que j'ai tort. Et je garde le droit de changer d'avis, ce qui après tout voudrait simplement dire rester créatif au-delà de 75 ans.» Well... même venant d'une éminente personnalité, n'est-ce pas là une pirouette?

1 Emanuel EJ. Why I Hope to Die at 75. Washington, D.C.: The Atlantic; October 2014; (article consultable en ligne).

2 «Pourquoi je souhaite mourir à 75 ans» (interview par P. Minet). Le Temps. 9 juin 2015; p. 14. 